\title{
Relationship Between Tactile Stimuli and Human Body Sway
}

\author{
Masaya Tadocoro, Taro Shibanoki \\ Ibaraki University, Hitachi, Ibaraki, Japan
}

\begin{abstract}
This paper describes the relationship between tactile stimulation and human body sway. We previously proposed a body sway mitigation system based on tactile stimulation and revealed that simultaneous stimulation behind both auricles were significantly improve human balance function, however, the mechanism of it were not fully discussed. In this paper, some stimulation patterns were applied to participants and COP distribution before/after stimulation was extracted. The results showed that COP values after stimulation could be inclined to the opposite of the stimulation site. It indicates that tactile stimuli can control human balance function.
\end{abstract}

Keywords: balance function, body sway, tactile stimulation, center of pressure

\section{Introduction}

According to a survey by the Tokyo Fire Department, falls account for about 80 percent of accidents ${ }^{1}$, and prevention of falls is an urgent issue in Japan, where the population is rapidly aging ${ }^{2}$.It is said that the decline of motor function with aging increases the risk of falls by about five times $^{3}$, and various balance function improvement and fall prevention systems have been developed $^{4-7}$

Studies focusing on improving balance function can be divided into two categories: those that use light touch contact (LTC) ${ }^{4}$ and those that use stimulation to the human body to improve vestibular and somatosensory fuctions $^{5-7}$. In LTC, it has been shown that the balance function can be improved by touching a fixed point. It has also been shown that it is possible to decrease and control body sway by applying acoustic ${ }^{5}$, electrical ${ }^{6}$ and vibratory $^{7}$ stimuli. However, because these approaches may exert a physical burden on the use, it is difficult to use in a daily life.

To overcome these problems, our research group has been proposed the body sway mitigation system based on a tactile stimulation and showed that body sway can be reduced via the application of vibratory stimuli around the pinna ${ }^{8}$. Here, acoustic stimulation can induce body sway to the opposite direction of the stimulus ${ }^{5}$, however,
Previous studies have not fully clarified the relationship between tactile stimulation and body sway.

In this paper, we attempt to quantify the influence of vibration stimulation on body sway by clarifying the relationship between vibratory stimulation near the auricle and body sway deflection. In the experiment, regular on-off stimulation patterns are applied to near the auricle of the subject, and the possibility of inducing center of pressure (COP) sway by the stimulation site is discussed.

\section{Method}

Figure 1 shows the proposed vibratory stimulationbalance function analysis method based on the body sway mitigation system using tactile stimulation ${ }^{8}$. The system consists of a stabilometer, tactile stimulators, a microcontroller for controlling vibratory stimulation pattern, and a PC. Stimulation patterns can be changed from the PC via TCP/IP communication. Figure 2 shows an example of stimulation pattern. The system can change the on-off interval $T_{S}[\mathrm{~s}]$, stimulation interval $T_{\text {on }}[\mathrm{s}]$, and non-stimulation interval $T_{\text {off }}[\mathrm{s}]$ of each tactile stimulator via PWM control. The details of the proposed vibratory stimulation-balance function analysis are shown below.

The subjects were asked to maintain an eye-closed tandem limb stance for $T[\mathrm{~s}]$. Tactile stimulators were 


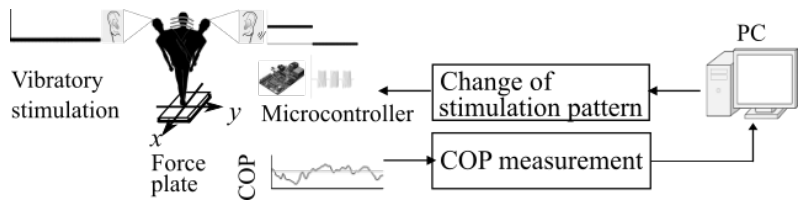

Fig. 1. Overview of the proposed balance function analysis system.

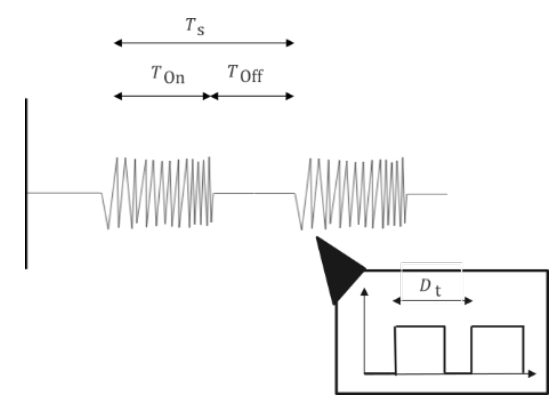

Fig. 2. An example of the stimulation pattern.

applied near the left and right auricles to provide vibration stimulation to the subjects. The stimulus intensity was the maximum stimulus intensity $D_{\max }$ during $T_{\text {on }}$ and $D_{\min }$ during $T_{\text {off }}$, which was less than or equal to the minimum stimulus intensity perceived by the subject.

In the experiment, the stimulus was applied to only one of the left and right sides. The time series waveform $\operatorname{COP}_{\{x, y\}}(t)$ of each axis of the measured center of pressure (COP) was smoothed by a second-order Butterworth digital low-pass filter (cut-off frequency: $f_{c}[\mathrm{~Hz}]$ ), and deviation from the median of each $T[\mathrm{~s}]$ was calculated. In this method, filtered COP value $\overline{\operatorname{COP}_{\{x, y\}}}(t)$ was divided into $M$ every $T_{s}[\mathrm{~s}]$, and the error between median values $\operatorname{med}_{m}$ in each interval $(m=1,2, \ldots, M)$ and mean values ave $e_{m}^{\text {on }}$ for stimulation interval and ave $\mathrm{off}_{m}^{\mathrm{of}}$ for non-stimulation interval were evaluated. This clarifies the bias of body sway with and without vibratory stimulation.

\section{Experiments}

\subsection{Experimental conditions}

In the experiments performed, COP sway during the application of vibratory stimulation was measured with one healthy male (23 [years]). Two motor oscillators (KD18B1) were connected to a Raspberry Pi 3B and a voltage was applied by pulse width control with a duty cycle of 0.1 [s]. The subject was asked to maintain tandem limb stance with eye-closed and the left leg back for $T=90[\mathrm{~s}]$ after a preparation time for $10[\mathrm{~s}]$. The COP was measured at a sampling frequency of $100[\mathrm{~Hz}]$ using a Wii Balance Board (Nintendo Co., Ltd.). The stimulus pattern applied to the subject was steady-state stimulus with $T_{s}=10[\mathrm{~s}]$, stimulus intensity $D_{\max }=$ $100[\%]$ during $T_{\text {on }}=5[\mathrm{~s}]$, and stimulus intensity $D_{\text {min }}=0[\%]$ during $T_{\text {off }}=5[\mathrm{~s}]$. This stimulus pattern was applied to left or right side.

The number of trials was twenty per side, and ten trials per side were conducted per day with an interval of one minute. The other ten trials were conducted on different days.

\subsection{Results and discussion}

Figure 3 (a) shows examples of signals measured during the experiments. Figure 3 (a) shows the results from the first trial, indicating stimulation patterns and COPs. The shaded areas represent times during nonstimulations. Figure 3 (b) is an enlargement of COPs from Fig. 3 (a), and it cannot be confirmed that the stimulus affected to the balance function.

Figure 4 (a) and (b) show histograms of COP of $y$ axis (frontal plane) with median and mean values for the leftside and right-side stimulus conditions, respectively. Figure 4(a) of the results for the left-side stimulus condition shows that the median is smaller than the mean value in the stimulus interval, and conversely, the median is larger than the mean value in the non-stimulus interval. In the Fig. 4, positive value shows deviation to the left 


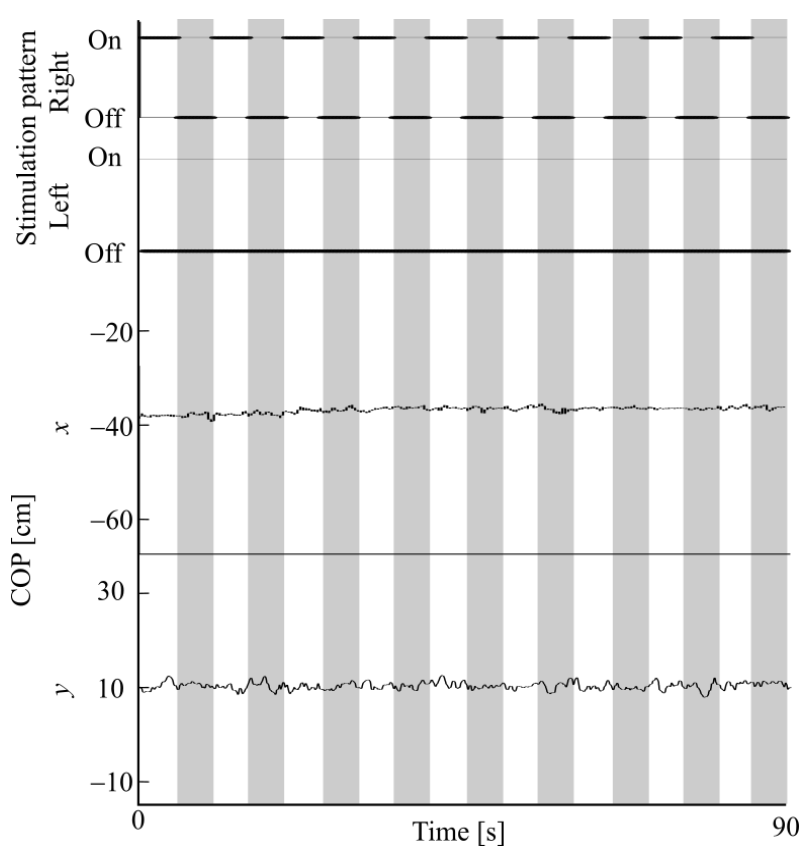

(a) $\mathrm{COP}$ in each direction

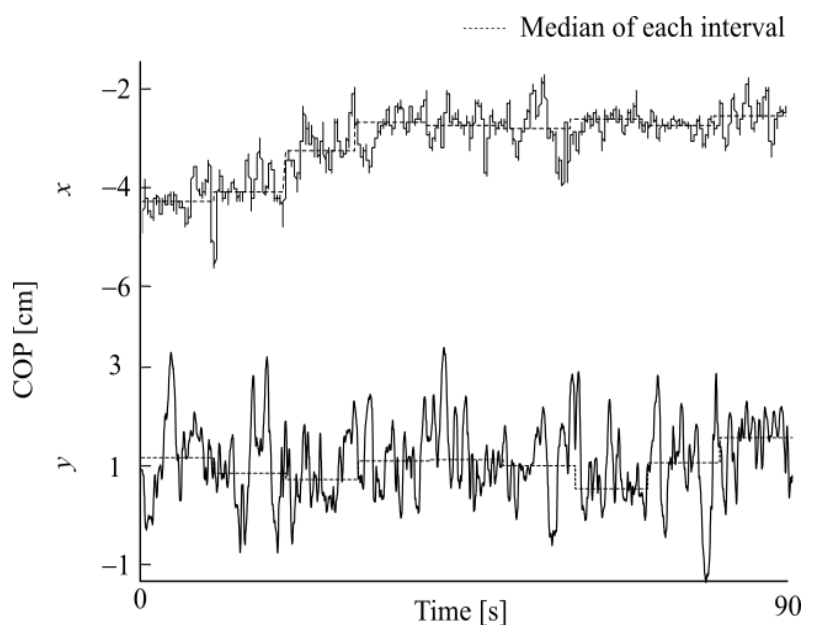

(b) An enlargement of COPs

Fig.3. Examples of experimental results.

side, therefore, the subject may tilt the opposite side of the stimulation.

Figure 5 shows the difference between mean values during stimulation and non-stimulation and the median in the interval of 10 [s] for (a) left-side stimulation condition and (b) right-side stimulation condition, respectively. The positive component represents the bias to the right side of COP distribution, and negative component represents the bias to the left side of COP

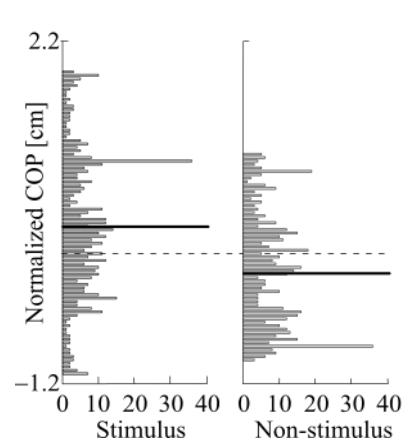

(a) Left stimulus

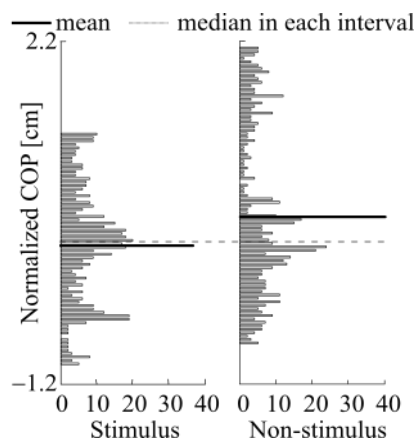

(b) Right stimulus
Fig. 4. Examples of COP distributions.

distribution. From the result, it was confirmed that the distribution of COP was deviated to the right during leftside stimulation and to the left during non-stimulation. The results of the heteroskedasticity $\mathrm{t}$-test confirmed a significant difference at the level of 0.1 [\%] (see Fig. 6 (a)). On the other hand, when right stimulation was applied, there was a bias to the left side during stimulation $(p<0.05)$, and a bias to the right side during non-stimulation $(\mathrm{p}<0.05)$ (see Fig. $6(\mathrm{~b})$. These results suggest that COP bias in the left-right direction may be induced to the opposite side of the stimulated side. It is possible that COP bias is induced to the opposite side of the stimulus, resulting in a larger bias toward the stimulus to maintain body balance equilibrium.

These results indicate that regular unilateral steady stimulation to the auricle induces COP sway on the opposite side of the stimulus, and that the sway may be deflected to the stimulus side to resist it. In the future, we will increase the number of subjects and investigate whether this tendency appears or not, and also whether it is possible to induce body sway arbitrarily by changing the vibration pattern or not.

\section{Conclusion}

In this paper, we examined the effects of regular unilateral steady-state stimulation patterns on COP bias when they were applied to near pinna. In the experiment, the subject was asked to maintain tandem limb positions with closed eyes, and the change of COP deviation with and without stimulation in each stimulation pattern was compared. It is confirmed that the stimulation may induce the deviation of COP oscillation in the frontal plane to the opposite side of the stimulated side. This 


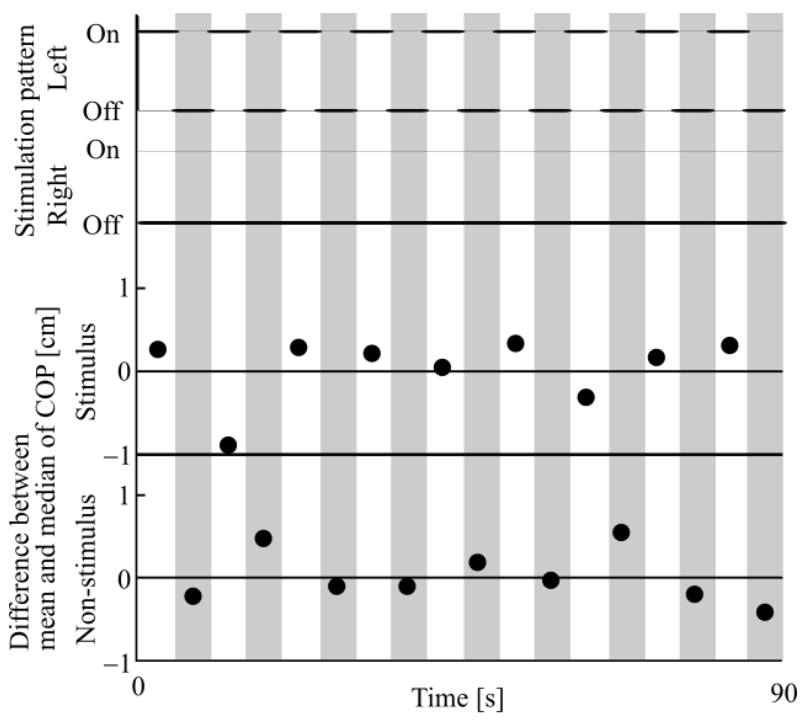

(a) Left stimulus

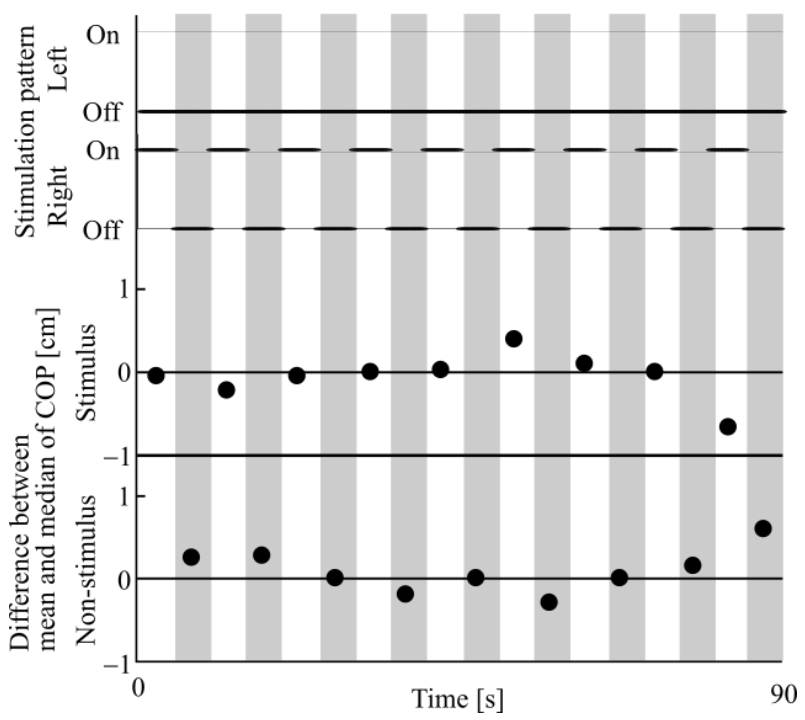

(b) Right stimulus

Fig. 5. The difference between the median and each mean value in the stimulation and non-stimulation intervals.

suggests that the amount of deflection to the stimulus side may increase to maintain the equilibrium against the induced COP oscillation.

In future research, we will increase the number of subjects and deepen the verification of the experiment conducted in this study. In addition, we plan to change the pattern of the vibratory stimulation and investigate the relationship with the body sway.

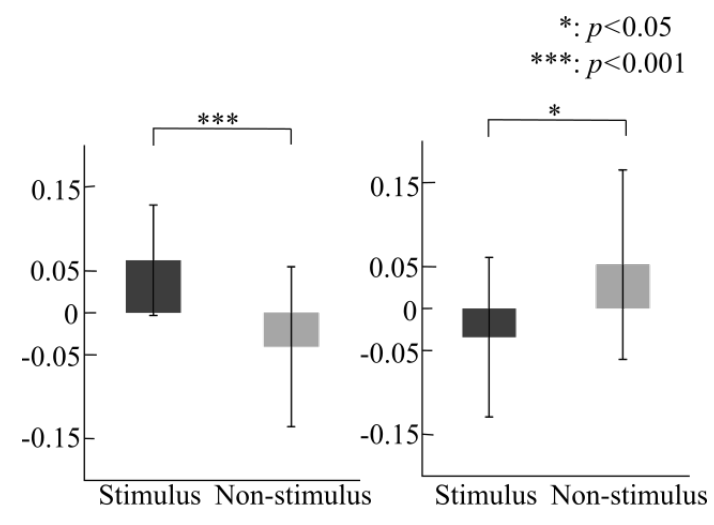

(a) Left stimulus

(b) Right stimulus

Fig. 6. Comparison of mean and median differences in COPs.

\section{Acknowledgements}

This work was supported by JSPS KAKENHI Grant Number 20K20212.

\section{References}

1. Tokyo Fire Department: https://www.tfd.metro.tokyo. lg.jp/lfe/topics/202009/kkhansoudeta.html

2. Statistics Bureau, Japan STATISTICAL HANDBOOK OF JAPAN 2020

3. Masdeu J.C., Sudarsky L., Wolfson L., "Gait Disorders of Aging: Falls and Therapeutic Strategies", Philadelphia, Lippincot -Raven, pp. 309-326, 1997

4. M.A. Riley, T.A. Stoffregen, M.J. Grocki, M.T. Turvey: Postural stabilization for the control of touching, Human Movement Science, Vol. 18, No. 6, pp. 795-817, 1999.

5. Miriam S. Welgampola and Brian L. Day, Craniocentric body-sway responses to $500 \mathrm{~Hz}$ bone-conducted tones in man, J Physiol 577.1, pp 81-95, 2006

6. Chisato Fujimoto, Yoshuharu Yamamoto, Teru Kamogashira, Makoto Kinoshita, Naoya Egami, Yukari Uemura, Fumiharu Togo, Tatsuya Yamasoba and Shinichi Iwasaki, Noisy galvanic vestibular stimulation induces a sustained improvedment in body balance in elderly adults, SCIENTIFIC REPORTS, 2016

7. W. Nanhoe-Mahabier, J.H. Allum, E.P. Pasman, S. Overem, B.R. Bloem, The effects of vibrotactile biofeedback training on trunk sway in Parkinson disease patients, Parkinsonism and Related Disorders Vol. 18, pp.1017-1021, 2012

8. Satowa Watanabe, Taro Shibanoki, Koji Shimatani, $A$ Body Sway Mitigration Method Based on Tactile Stimulation, Proceedings of the SICE Annual Conference 2016, pp.518-19, 2016 